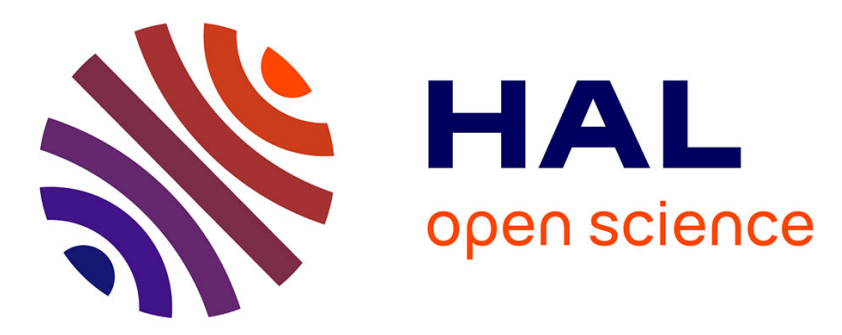

\title{
Modeling and Stress Analysis of a Pre-Shaped Curved Beam : Influence of High Modes of Buckling
}

Hussein Hussein, Patrice Moal, Gilles Bourbon, Yassine Haddab, Philippe Lutz

\section{- To cite this version:}

Hussein Hussein, Patrice Moal, Gilles Bourbon, Yassine Haddab, Philippe Lutz. Modeling and Stress Analysis of a Pre-Shaped Curved Beam : Influence of High Modes of Buckling. International Journal of Applied Mechanics, 2015, 7 (4), 20 p. hal-01303387

\section{HAL Id: hal-01303387 \\ https://hal.science/hal-01303387}

Submitted on 19 May 2016

HAL is a multi-disciplinary open access archive for the deposit and dissemination of scientific research documents, whether they are published or not. The documents may come from teaching and research institutions in France or abroad, or from public or private research centers.
L'archive ouverte pluridisciplinaire HAL, est destinée au dépôt et à la diffusion de documents scientifiques de niveau recherche, publiés ou non, émanant des établissements d'enseignement et de recherche français ou étrangers, des laboratoires publics ou privés. 


\title{
Modeling and Stress Analysis of a Pre-Shaped Curved Beam: Influence of High Modes of Buckling
}

\author{
Hussein Hussein*, Patrice Le Moal ${ }^{\dagger}$, Gilles Bourbon ${ }^{\ddagger}$, \\ Yassine Haddab ${ }^{\S}$ and Philippe Lutz ${ }^{\uparrow}$ \\ FEMTO-ST, Univ. Bourgogne Franche-Comté \\ Univ. de Franche-Comté/CNRS/ENSMM, Besançon, France \\ *hussein.hussein@femto-st.fr \\ ${ }^{\dagger}$ patrice.lemoal@femto-st.fr \\ ${ }^{\ddagger}$ gilles.bourbon@femto-st.fr \\ §yassine.haddab@femto-st.fr \\ 『philippe.lutz@femto-st.fr
}

\begin{abstract}
In this paper, we investigate the effect of high modes of buckling on the mechanical behavior of a pre-shaped curved beam. In a first stage, the presented modeling develops further the snapping forces solution and bistability conditions in order to include high modes of buckling. In a second stage, we develop the analytical solution of the stresses inside the beam during deflection between the two sides of buckling. The buckling with or without mechanical conditions on antisymmetric modes, the force characteristics, bistability conditions and stresses are described in this paper based on mathematical approach in order to provide a clear physical understanding of the curved beam behavior and its design parameters. The accurate knowledge of the design parameters is important in order to achieve the best integration of the curved beam in a complete microstructure. The analytical results are compared with and without considering high modes of buckling and have shown to be in excellent agreement with FEM simulations. The results show the importance of the high modes in calculating stresses and snapping forces.
\end{abstract}

Keywords: Pre-shaped curved beam; buckling; bistable mechanism; stress state; MEMS.

\section{Introduction}

In microsystems, numerous applications can benefit from bistability, such as switches, valves, relays, positioners [Chen et al., 2011], braille displays [Niu et al., 2012], reconfigurable robotic devices [Hafez et al., 2003] and digital microrobotics. [Chen et al., 2008, 2010; Chalvet et al., 2011, 2013]. Bistable structures are generally simple and allow assigning a binary state to every reachable position.

The stability of a bistable system can be ensured either by active or passive maintain. In active maintain [Fukuta et al., 2006; Abadie et al., 2009; Driesen et al., 2010], the mechanism needs some external energy to keep its place, while 
it is not the case in passive maintain where the mechanism is inherently stable at each position. Generally, passive maintain has many advantages such as low energy consumption due to short actuation time and self-stability which allows improving the repeatability with an open loop control. Many passive maintain examples can be found in literature, we can mention magnetic maintain [Zhang et al., 2007; Wu et al., 2010; Barth and Kohl, 2010], bistable electro active polymers [Yu et al., 2010], and compliant mechanisms [Oberhammer et al., 2006; Chen et al., 2009a,b, 2010; Huang and Yang, 2013].

Further, compliant mechanisms exhibit many advantages such as increased precision and reliability, no friction, reduced wear and low manufacturing costs.

Curved beam can be considered as a bistable system that combines the advantages of passive maintain and compliant mechanisms.

In this paper, we develop the analytical solution of the snapping forces and stresses with considering high modes of buckling. The impact of high modes of buckling on the snapping forces, the bistable behavior and the stress state is discussed based on a mathematical approach.

Bistable curved beam designs are still paying the price for the lack of physical intuition by resorting to the FEM modeling, where there is a difficulty to consider all complex and nonlinear effects. Euler elastica theory was the starting point to study the beam buckling for large deformation. Based on Lagrangian approach, Vangbo [1998] carried out one of the first studies on pre-compressed curved beams that takes compressibility into account for small deformations. The obtained expressions consider high modes of buckling. Buckling behavior of a suspended microbeam as a result of electrothermal expansion is investigated by Chiao and Lin [2000]. Emam and Nayfeh examine in their studies [Emam and Nayfeh, 2004; Nayfeh and Emam, 2008] the vibration and dynamics of postbuckling configurations of a beam. Park and Hah [2008] demonstrate that bistability of buckled beams depends not only on a ratio of its initial rise to its thickness, but also on its residual stress. Cazottes [2009] in his thesis has studied the bistability of a pre-compressed curved beam when it is actuated either by force or by moment. Elastica models for static and dynamic analysis with solutions and experiments are given by Camescasse [2013] in his thesis. Chen et al. [Chen and Hung, 2012; Chen and Tsao, 2013], carried out one of the last studies on extensible elastica theory of a curved beam.

Concerning the case of a pre-shaped curved beam, Qiu et al. [2004] have calculated analytically the snapping forces by neglecting high modes of buckling and they provide an approximation of the solution with high modes for high values of the initial height-to-thickness ratio. Their analytical results are approved by FEM simulations and experiments.

This paper recalls in a first stage the solution of the snapping forces of a preshaped curved beam with clamped-clamped boundary conditions. The solution is obtained in Qiu et al. [2004] using an approximation of neglecting high modes of buckling. 
In Sec. 3, we develop analytically the solution of the snapping forces with considering high modes of buckling. The analytical expressions obtained with high modes implicitly include the effects of all modes of buckling on the curved beam behavior. Existence and magnitude of bistability are discussed with respect to the snapping forces evolution.

In Sec. 4, evolution of stresses inside the curved beam is calculated analytically with and without the approximation of high modes. Calculating the stress state is important for the design, particularly for miniaturization and optimization purposes under elastic or failure limits.

Finally, the analytical results with and without high modes of buckling are compared with FEM simulations. This comparison shows the interest to consider high modes of buckling in the modeling of the curved beam behavior.

\section{Buckling of a Beam}

\subsection{Buckled beam model}

Buckling a beam is defined as a sudden deformation which occurs when the excess of compression energy stored in the beam is converted into bending energy. In other words, transverse deflection occurs when the compressive force $P$ exceeds a critical value $P_{0}$, the beam enters in the first buckling mode (Fig. 1).

The postbuckling configuration of the beam can be considered as a compliant mechanism that shows bistability. The buckled beam shows stability of its position at two possible configurations which are symmetrically distributed in the two buckling sides (Fig. 2).
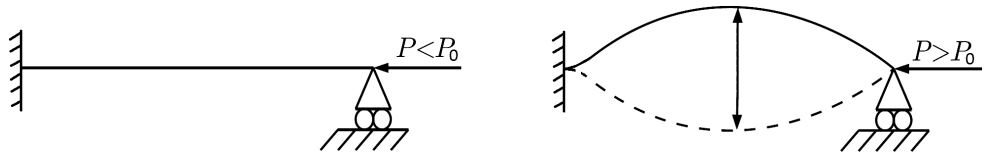

Fig. 1. Buckling of a beam before and after a critical axial compression.
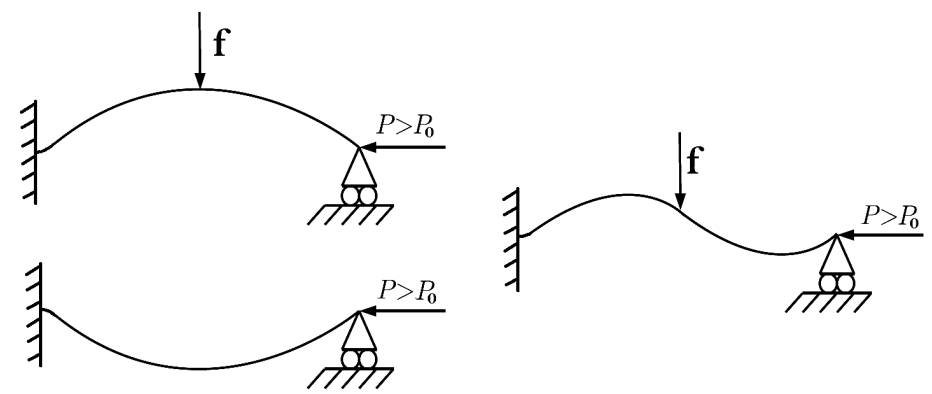

Fig. 2. Transition between the two stable positions of a buckled beam as a result of a lateral force applied in the middle of the beam. 
In most cases, and in this paper, the action can be a lateral force applied in the middle of the beam. Otherwise, the action can be also a force applied in different points [Camescasse, 2013], an electromagnetic field [Park and Hah, 2008], an electrostatic field [Krylov and Dick, 2010; Tajaddodianfar et al., 2014] or moments applied in determined locations on the beam [Stoimenov et al., 2007; Cazottes, 2009].

There are two possible approaches to deal with the post-buckling problem, based on static and dynamic models. In dynamic modeling, there are two types of modes, buckling modes that depend on axial stress and resonance modes that depend on the system frequency. In contrast, since it does not consider the time, static modeling exhibits only buckling modes.

Generally, the resonance frequency of a clamped-clamped pre-shaped curved beam is higher while reducing the beam dimensions. MEMS devices generally range in size from $20 \mu \mathrm{m}$ to few millimeters. An interest to dynamic modeling can be referred to studies in Emam and Nayfeh [2004], Nayfeh and Emam [2008], Cazottes [2009], Camescasse [2013], Tajaddodianfar et al. [2014]. In this study, we investigate the static modeling.

Curved beams can be divided on the basis of the fabrication process to three kinds [Zaidi et al., 2011]:

- Pre-compressed curved beams where the beam is fabricated rectilinear then it is compressed [Vangbo, 1998; Cazottes, 2009; Camescasse, 2013].

- Pre-stressed curved beams where during fabrication, the beam is buckled due to residual stress which is added by heating or oxidation [Pane and Asano, 2008].

- Pre-shaped curved beams where the beam is directly fabricated at the first buckling shape mode without residual stresses [Qiu et al., 2001].

The behavior of the pre-compressed curved beam is symmetrical between the two sides of buckling, in terms of the snapping force, beam shape and stresses evolution. However, in the case of microfabrication, the monolithic constraint makes this solution difficult to use. The pre-stressed curved beam makes the integration into a monolithic device easier, but the residual stress is difficult to control by fabrication [Zaidi et al., 2011]. However, for the pre-shaped curved beam, despite the fact that symmetry is lost and that bistability exists under some conditions, fabrication and integration are simpler. Some solutions are presented in the literature to improve the symmetry property of pre-shaped curved beams [Jensen et al., 2001; Qiu et al., 2004].

In this paper, we investigate the modeling of a pre-shaped curved beam fabricated directly at the first buckling shape mode. The model of the curved beam presented in Fig. 3 has the following characteristics: axial force $P$, thickness $t$, depth $b$, span $l$, deflection $d$, beam shape $w(x)$ and applied lateral force in the middle of span $f$. Moreover, $h$ is the height of the fabricated curved beam at $x=\frac{l}{2}$. 


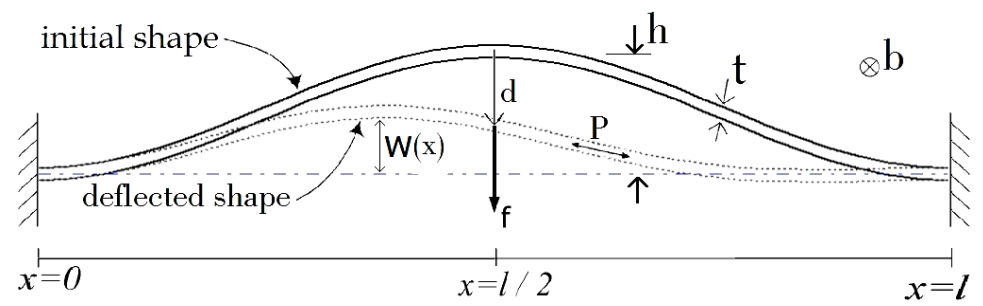

Fig. 3. Clamped-clamped curved bistable beam at initial position and after deflection.

\subsection{Buckling equation}

Based on small deformation hypothesis, Timoshenko (1961) gives the buckling equation of a curved beam:

$$
\frac{\mathrm{d}^{2}}{\mathrm{~d} x^{2}}\left(E I \frac{\mathrm{d}^{2} w}{\mathrm{~d} x^{2}}\right)+P \frac{\mathrm{d}^{2} w}{\mathrm{~d} x^{2}}=0
$$

where $E$ is the Young's modulus and $I=\iint z^{2} \mathrm{dydz}$ is the quadratic moment.

Considering a uniform beam in terms of material properties and sections:

$$
E I \frac{\mathrm{d}^{4} w}{\mathrm{~d} x^{4}}+P \frac{\mathrm{d}^{2} w}{\mathrm{~d} x^{2}}=0
$$

Introducing the clamped-clamped boundary conditions implies the modal nature of the solution. The solution is an infinite sum of buckling modes:

$$
w(x)=\sum_{j=1}^{\infty} a_{j} w_{j}(x)
$$

where $a_{j}$ is the $j$ th constant mode which reflects the contribution of each buckling mode in the total solution, and $w_{j}(x)$ is the $j$ th buckling shape mode:

$$
\left.\begin{array}{r}
w_{j}=1-\cos N_{j} \frac{x}{l}, \\
N_{j}=(j+1) \pi,
\end{array}\right\} \quad j=1,3,5, \ldots,
$$

where $N_{j}$ is the $j$ th mode of the normalized axial force $N$ and $N=\sqrt{\frac{P l^{2}}{E I}}$.

\subsection{Snapping forces without high modes of buckling}

The approximation of neglecting high modes of buckling simplifies the calculation [Qiu et al., 2004]. Normalization is taken in order to simplify the solution:

$X=\frac{x}{l} ; \quad W_{j}(X)=w_{j}(x) ; \quad W(X)=\frac{w(x)}{h}=\sum_{j=1}^{\infty} A_{j} W_{j}(X) ; \quad F=\frac{f l^{3}}{E I h} ; \quad \Delta=\frac{d}{h}$. 


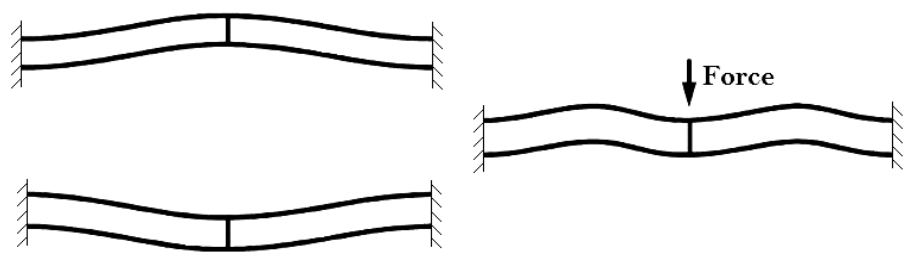

Fig. 4. Transition between the two stable positions of two curved beams connected in the middle, mode 3 appears during transition.

The variational calculation in Qiu et al. [2004] leads to three kinds of solutions. The first kind is when the curved beam is in the compressible phase:

$$
\left\{\begin{array}{l}
F=F_{1}, \\
N<\left\{\begin{array}{l}
N_{2} \text { mode } 2 \text { is not constrained, } \\
N_{3} \text { mode } 2 \text { is constrained, }
\end{array}\right. \\
A_{j} \neq 0 ; \quad j=1,5,9,13, \ldots
\end{array}\right.
$$

The second kind is when $N$ reaches $N_{2}$ without mechanical constraints:

$$
\left\{\begin{array}{l}
F=F_{2}, \\
N=N_{2}, \\
A_{j} \neq 0 ; \quad j=1,2,5,9,13, \ldots
\end{array}\right.
$$

The third kind is when mode 2 is constrained and $N$ reaches $N_{3}$ :

$$
\left\{\begin{aligned}
F & =F_{3}, \\
N & =N_{3}, \\
A_{j} \neq 0 ; \quad j & =1,3,5,9,13, \ldots
\end{aligned}\right.
$$

Mode 2 is constrained by connecting two curved beams in the middle as shown in Fig. 4. Otherwise, for a simple curved beam without constraints, the stability margin for the second stable position is too low. In this paper, we are interested only to the first and third kinds of solutions. The results for the second kind are similar to those of the third kind in calculation.

The terms $A_{j}$, for $j=1,5,9,13, \ldots$ have the following values:

$$
\begin{aligned}
& A_{1}=-\frac{1}{2} \frac{N_{1}^{2}}{N^{2}-N_{1}^{2}}+\frac{4 F}{N_{1}^{2}\left(N^{2}-N_{1}^{2}\right)}, \\
& A_{j}=\frac{4 F}{N_{j}^{2}\left(N^{2}-N_{j}^{2}\right)} \text { for } j=5,9,13, \ldots
\end{aligned}
$$

Two characteristics equations that describe the curved beam behavior are derived from the variational calculation:

$$
\begin{aligned}
\Delta & =1-2 \sum_{j=1,5,9, \ldots} A_{j}, \\
\frac{N^{2}}{12 Q^{2}} & =\frac{N_{1}^{2}}{16}-\sum_{j=1}^{\infty} \frac{A_{j}^{2} N_{j}^{2}}{4},
\end{aligned}
$$

where $Q$ is the height-to-thickness ratio $Q=h / t$. 
The following equations summarize the first and third kinds of solution the snapping forces that are calculated in Qiu et al. [2004] without high modes of buckling. For the first kind:

$$
\left\{\begin{array}{l}
F_{1}=\frac{3 \pi^{4} Q^{2}}{2} \Delta\left(\Delta^{2}-3 \Delta+2+\frac{4}{3 Q^{2}}\right), \\
N^{2}=3 \pi^{2} Q^{2}\left(-\Delta^{2}+2 \Delta\right), \\
W(X)=A_{1} W_{1}(X) ; \quad A_{1}=\frac{1-\Delta}{2} .
\end{array}\right.
$$

For the third kind:

$$
\left\{\begin{array}{l}
F_{3}=6 \pi^{4}\left(\frac{4}{3}-\Delta\right) \\
N=N_{3} \\
W(X)=A_{1} W_{1}(X)+A_{3} W_{3}(X) \\
A_{1}=\frac{1-\Delta}{2} ; \quad A_{3}^{2}=-\frac{1}{16}\left(\Delta^{2}-2 \Delta+\frac{16}{3 Q^{2}}\right) .
\end{array}\right.
$$

\section{Snapping Forces with High Modes of Buckling}

In this section, we develop the solution of the snapping forces taking into account the high modes of buckling. The stability equilibrium is then analyzed in the two sides of buckling based on the snapping forces solution.

\subsection{Snapping forces}

Combining Eqs. (9) and (10) with (11) and (12), the two infinite series become:

$$
\begin{aligned}
& \sum_{j=1,5,9, \ldots} A_{j}=-\frac{1}{2} \frac{N_{1}^{2}}{N^{2}-N_{1}^{2}}+4 F \cdot \mathrm{Sum}_{1}, \\
& \sum_{j=1}^{\infty} A_{j}^{2} N_{j}^{2}=\frac{1}{4} \frac{N_{1}^{2}\left(N_{1}^{4}-16 F\right)}{\left(N^{2}-N_{1}^{2}\right)^{2}}+16 F^{2} \cdot \mathrm{Sum}_{2},
\end{aligned}
$$

where $\mathrm{Sum}_{1}$ and $\mathrm{Sum}_{2}$ have the following shapes:

$$
\begin{aligned}
\operatorname{Sum}_{1} & =\sum_{j=1,5,9, \ldots}^{\infty} \frac{1}{N_{j}^{2}\left(N^{2}-N_{j}^{2}\right)}, \\
\operatorname{Sum}_{2} & =\sum_{j=1,5,9, \ldots}^{\infty} \frac{1}{N_{j}^{2}\left(N^{2}-N_{j}^{2}\right)^{2}} .
\end{aligned}
$$

Imposing $j=4 k+1, \operatorname{Sum}_{1}$ in (17) can be decomposed in two infinite sums:

$$
\frac{1}{4 \pi^{2} N^{2}}\left[\sum_{k=0}^{\infty} \frac{1}{(2 k+1)^{2}}-\sum_{k=0}^{\infty} \frac{1}{(2 k+1)^{2}-\left(\frac{N}{2 \pi}\right)^{2}}\right] .
$$


The first Sum in (19) is equal to:

$$
\sum_{k=0}^{\infty} \frac{1}{(2 k+1)^{2}}=\frac{\pi^{2}}{8}
$$

The second Sum can be concluded from the following equation [Remmert, 1991]:

$$
\pi \tan \left(\frac{\pi}{2} x\right)=\sum_{k=0}^{\infty} \frac{4 x}{(2 k+1)^{2}-x^{2}} .
$$

Then, $\mathrm{Sum}_{1}$ is equal to:

$$
\operatorname{Sum}_{1}=\frac{1}{8 N^{3}}\left[\frac{N}{4}-\tan \left(\frac{N}{4}\right)\right] .
$$

Introducing (22) in (11), a new equation is derived:

$$
F=\frac{N^{3}}{\frac{N}{4}-\tan \frac{N}{4}}\left(\frac{N^{2}}{N^{2}-4 \pi^{2}}-\Delta\right) .
$$

The sum in (18) can be obtained by deriving the sum in (17) with respect to $N$ :

$$
\frac{\partial\left(\operatorname{Sum}_{1}\right)}{\partial N}=-2 N \cdot \mathrm{Sum}_{2}
$$

Then, $\mathrm{Sum}_{2}$ is equivalent to:

$$
\operatorname{Sum}_{2}=\frac{3}{64 N^{4}}\left[1-\frac{\tan \left(\frac{N}{4}\right)}{\frac{N}{4}}+\frac{\tan ^{2}\left(\frac{N}{4}\right)}{3}\right] .
$$

Introducing (25) in (12), the following equation is obtained for the first kind of solution:

$\frac{3}{16 N^{4}}\left(1+\frac{\tan ^{2} \frac{N}{4}}{3}-\frac{\tan \frac{N}{4}}{\frac{N}{4}}\right) F_{1}^{2}-\frac{4 \pi^{2}}{\left(N^{2}-4 \pi^{2}\right)^{2}} F_{1}+\frac{N^{2}}{12 Q^{2}}-\frac{\pi^{2} N^{2}\left(N^{2}-8 \pi^{2}\right)}{4\left(N^{2}-4 \pi^{2}\right)^{2}}=0$.

Equations (23) and (26) are the characteristic equations which allow defining the relations between $F, N$ and $\Delta$ for the first kind of solution. In this case, the problem can be solved by numerical method. The idea is to change $N$ in (26) from 0 to the point where there are no real solutions. Two values of $F$ are obtained for each value of $N$. Introducing these values in (23), the relations $N-\Delta$ and $F-\Delta$ are obtained.

Figure 5 shows evolution of $N$ in function of $\Delta$ for different values of $Q$. Shapes of the curved beam during snapping between two sides of buckling are shown in Figure 5 with first, second and third kinds of solution. Noting that the normalized axial force $N$ is equivalent to zero at $(\Delta=0, F=0)$ and at $\left(\Delta=20 / \pi^{2}, F=\right.$ $\left.3840 / \pi^{2}\right)$. The normalized displacement at the end of deflection is close to 2 , but not exactly as it is for the pre-compressed beam. 


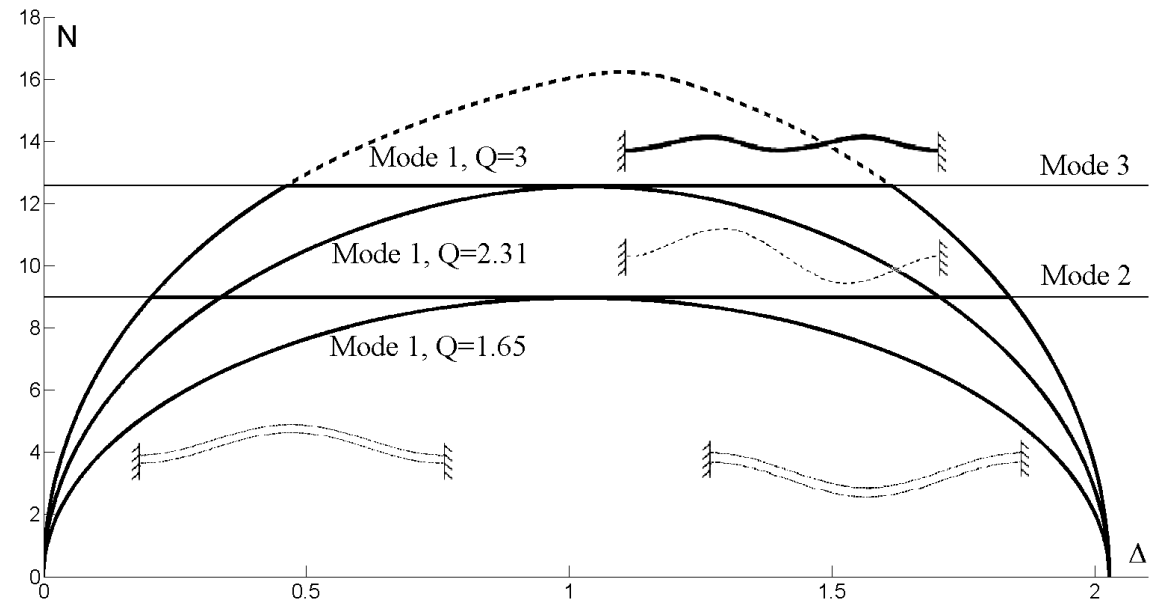

Fig. 5. Evolution of the normalized axial force $N$ in the first kind of solution in function of $Q$ ratio. $N$ is constant in the second and third kinds of solution. The shape of the curved beam in the first, second and third case.

Furthermore, Fig. 5 illustrates the values of $Q$ providing the transitions between the first, second and third kinds of solution. In the first kind of solution, the maximum value of $N$ that can be reached during snapping increases with increasing the value of $Q . N$ exceeds $N_{1}$ only for:

$$
Q \geq \sqrt{\frac{64 \pi^{2}}{117-7 \pi^{2}}} \approx 1.16 .
$$

Noting that at $N=N_{1}$, the normalized force has a unique value $F=2 \pi^{4}$.

On the other side, the third kind of solution is simpler. Making $N$ constant at $N_{3}=4 \pi$, the third kind simplify the previous equations. Evolution of the force can be directly concluded from (23):

$$
F_{3}=64 \pi^{2}\left(\frac{4}{3}-\Delta\right)
$$

Equation (28) exhibits a perfect linear interaction between force and displacement in the third kind of solutions. Figure 6 shows evolution of $F$ in function of $\Delta$ for different values of $Q$.

On the other side, the mode constant $A_{3}$ which appears in the third kind of solution is obtained by recalculating (26) without canceling $A_{3}$, then introducing (23) in the new equation:

$$
A_{3}^{2}=-\frac{3}{4 \pi^{2}} \Delta^{2}+\frac{14}{9 \pi^{2}} \Delta+\frac{1}{18}-\frac{20}{27 \pi^{2}}-\frac{1}{3 Q^{2}} .
$$

Noting that the sign of $A_{3}$ changes with the direction of deflection. 


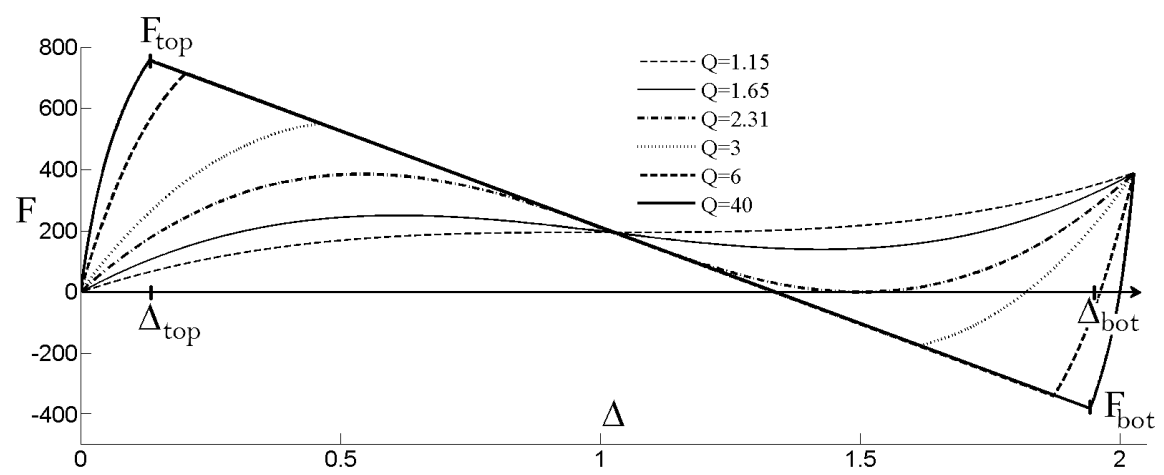

Fig. 6. Evolution of the normalized snapping force $F$ of the pre-shaped curved beam for different $Q$ values when mode 2 is constrained.

In light of the equation of $A_{3}$, the third mode cannot appear unless $Q$ respects the following condition:

$$
Q \geq \sqrt{\frac{162 \pi^{2}}{27 \pi^{2}+32}} \approx 2.314
$$

Further, $\Delta_{\text {top }}$ and $\Delta_{\text {bot }}$ (Fig. 6), which are the exact positions where the third mode appears, can be concluded from (29):

$$
\Delta_{\text {top }}, \Delta_{\text {bot }}=\frac{28}{27} \pm \frac{2 \pi}{3} \sqrt{\frac{1}{6}+\frac{16}{81 \pi^{2}}-\frac{1}{Q^{2}}} .
$$

The exact values of $F_{\text {top }}$ and $F_{\text {bot }}$ (Fig. 6) can be concluded simply by introducing the values of $\Delta_{\text {top }}$ and $\Delta_{\text {bot }}$, respectively in (28).

\subsection{Bistability conditions}

Physically, the curves in Fig. 6 present the amount of the lateral force produced by the beam in the center point after displacement. In this context, the bistability that we look for is provided by the negative portions of $F$ produced by the beam that will push to the other buckling side.

As we can conclude from Fig. 6, the value of the snapping force is not symmetric between the two sides of buckling. This comes from the bending energy which starts its evolution from the initial shape per fabrication.

The shift-up of the curves in Fig. 6 affects the bistability behavior. Mechanical conditions must be considered in order to involve bistability, while the greater margin of stability remains in the first side of buckling.

The stable positions are the points where $F$ is equivalent to zero and the beam tends to return to its position after a small displacement. Thus, canceling $F$ in $(26)$ for the first kind of solution, three values of $N$ are obtained. Putting these values 
in (23), three values of $\Delta$ are obtained:

$$
\Delta=\left\{0, \frac{3}{2} \pm \sqrt{\frac{1}{4}-\frac{4}{3 Q^{2}}}\right\} .
$$

We conclude from (32) and Fig. 6 that the beam exhibits two stable positions, only for $Q>\sqrt{\frac{16}{3}} \approx 2.31$. The second $\Delta$ is unstable position because every variation of its state will create a tendency to move away.

Moreover, due to mode 3 , when $Q \geq \sqrt{6} \approx 2.45$, there are three new values of $\Delta$ where $F$ is equal to zero:

$$
\Delta=\left\{0, \frac{4}{3}, \frac{3}{2}+\sqrt{\frac{1}{4}-\frac{4}{3 Q^{2}}}\right\} .
$$

The first and last $\Delta$ values in (33) are the two stable points. In the other side, the $\Delta$ positions which cancel $F$ for the second kind of solution are:

$$
\Delta=\left\{0,1.96, \frac{3}{2}+\sqrt{\frac{1}{4}-\frac{4}{3 Q^{2}}}\right\} .
$$

A curved beam where the unsymmetrical modes of buckling are not constrained (including mode 2) will never show bistability unless $Q>5.65$. The bistability in this case is very limited and no important force is obtained in the second side of buckling.

Table 1 summarizes the conditions on $Q$ in order to reach $N_{1}, N_{2}, N_{3}$ and the bistability feature. The normalized axial force $N$ inside the curved beam reaches $N_{1}$ during deflection between the two sides of buckling only for $Q>1.16$ and reaches $N_{2}$ for $Q>1.65$ when mode 2 is not constrained and reaches $N_{3}$ for $Q>2.31$ when mode 2 is constrained. The bistability exists only for $Q>5.65$ when mode 2 is not constrained and for $Q>2.31$ when mode 2 is constrained.

\section{Stress State}

In this section, axial and bending stresses are calculated with and without the approximation of high modes. This allows obtaining the evolution of the maximal total stress inside the curved beam during deflection.

Table 1. Conditions on $Q$ in order to reach modes 1-3 and the bistability feature for the curved beam.

\begin{tabular}{lcc}
\hline & $\begin{array}{c}\text { Mode 2 } \\
\text { unconstrained }\end{array}$ & $\begin{array}{c}\text { Mode 2 } \\
\text { constrained }\end{array}$ \\
\hline Mode 1 & $Q \geq 1.16$ & $Q \geq 1.16$ \\
Mode 2 & $Q \geq 1.65$ & does not appear \\
Mode 3 & does not appear & $Q \geq 2.31$ \\
Bistability & $Q \geq 5.65$ & $Q \geq 2.31$ \\
\hline
\end{tabular}




\subsection{Stresses without high modes}

Stresses inside the beam are decomposed into axial stress and bending stress. Axial stress is constant along the beam and has a maximum when the deflection is around the middle while bending stress changes along the beam sections and increases as far as the deflection is closer to the other side. The axial stress $p$ is equivalent to:

$$
p=\frac{E t^{2}}{12 l^{2}} N^{2} .
$$

The bending stress $T$ starts evolution from the initial shape:

$$
T=E z\left(\frac{\mathrm{d}^{2} \bar{w}}{\mathrm{~d} x^{2}}-\frac{\mathrm{d}^{2} w}{\mathrm{~d} x^{2}}\right),
$$

where $\bar{w}(x)$ is the initial shape of the beam and $z$ is the distance in the cross-section to the neutral line.

For the first kind of solution, axial stress is simply concluded from (13) and (35):

$$
p=\pi^{2} \frac{E t h}{l^{2}} \frac{Q}{4}\left(-\Delta^{2}+2 \Delta\right)
$$

Also, bending stress is obtained using (13) and (36):

$$
T=\frac{2 \pi^{2} E z h}{l^{2}} \Delta \cos 2 \pi \frac{x}{l}
$$

The same for the third kind of solution, axial and bending stresses are calculated from (14), (35) and (36):

$$
\begin{aligned}
p & =\pi^{2} \frac{E t h}{l^{2}} \frac{4}{3 Q}, \\
T & =\frac{2 \pi^{2} E z h}{l^{2}}\left(\Delta \cos 2 \pi \frac{x}{l}-2 \sqrt{-\Delta^{2}+2 \Delta-\frac{16}{3 Q^{2}}} \cos 4 \pi \frac{x}{l}\right) .
\end{aligned}
$$

The total stress inside the beam is simply the sum of the axial and bending stresses. However, analyzing (38) and (40), the extremum of the bending stress is noticed at the midpoint $x=\frac{l}{2}$ and at boundaries $x=[0, l]$ when the first kind of solution is present and only at the middle when mode 3 is present. Also, the stress is maximized when $z$ is at the limits $z=\left|\frac{t}{2}\right|$. Then, putting these values in the stress equations, the absolute value of the maximal total stress during deflection can be written as follows, for the first kind of solution:

$$
\sigma_{\max }^{\Delta}=\pi^{2} \frac{E t h}{l^{2}}\left(-\frac{Q}{4} \Delta^{2}+\left(1+\frac{Q}{2}\right) \Delta\right) .
$$

And for the third kind of solution:

$$
\sigma_{\max }^{\Delta}=\pi^{2} \frac{E t h}{l^{2}}\left(\Delta+2 \sqrt{-\Delta^{2}+2 \Delta-\frac{16}{3 Q^{2}}}+\frac{4}{3 Q}\right) .
$$

Analyzing the last two equations, we notice the presence of three different forms of evolution curves for the maximal stress during deflection, as shown in Fig. 7. 


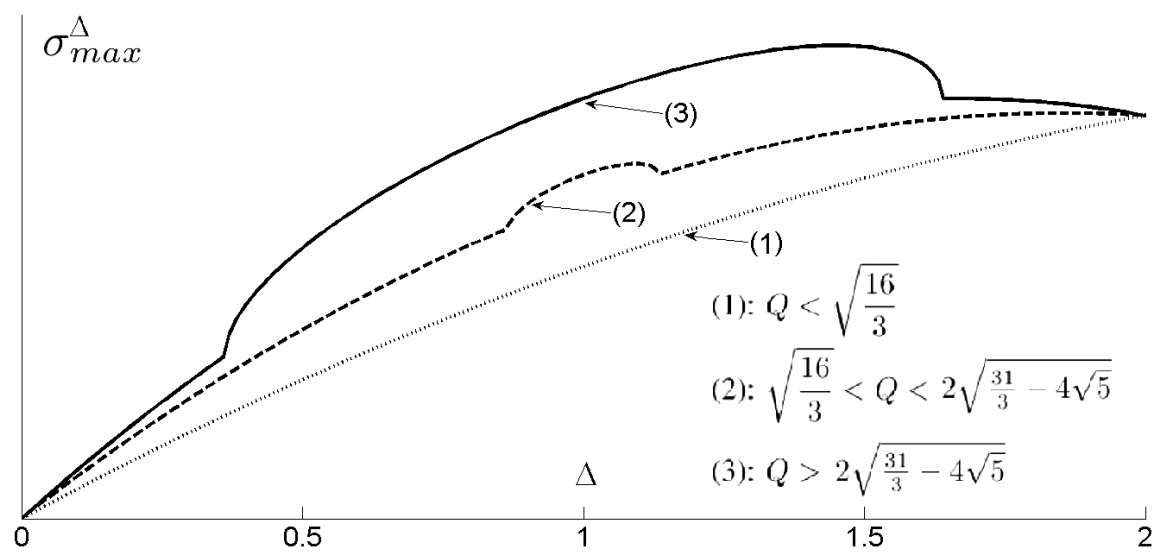

Fig. 7. Evolution of the maximal stress in the beam during deflection for different values of $Q$.

The first form of stress is when only the first kind of solution exists. The second form is when the third kind of solution appears during deflection. The third form is when the maximal stress point is higher in the third kind of solution domain.

The first form exists for $Q<\sqrt{\frac{16}{3}} \approx 2.31$, while the third one appears for $Q>2$ $\sqrt{\frac{31}{3}-4 \sqrt{5}} \approx 2.36$. The second form of stress exists between the last two values of $Q$. Noting that there is a small difference between the last two values of $Q$, which means that the second form of stress is a rare case.

The maximal stress point in the first two forms is at $\Delta=2$ when $Q \leq 2$ and at $\Delta=1+2 / Q$ when $Q \geq 2$. Thus, for $Q \leq 2$, the maximal stress $\sigma_{\max }$ is equivalent to:

$$
\sigma_{\max }=2 \pi^{2} \frac{E t h}{l^{2}}
$$

When $Q$ is between $[2 ; 2.36], \sigma_{\max }$ is equivalent to:

$$
\sigma_{\max }=\pi^{2} \frac{E t h}{l^{2}}\left(1+\frac{1}{Q}+\frac{Q}{4}\right) .
$$

In the third form, the maximal stress point is at:

$$
\Delta=1+\frac{1}{\sqrt{5}} \sqrt{1-\frac{16}{3 Q^{2}}} .
$$

Introducing this value in $(42)$, for $Q>2.36, \sigma_{\max }$ is equivalent to:

$$
\sigma_{\max }=\pi^{2} \frac{E t h}{l^{2}}\left(1+\frac{4}{3 Q}+\sqrt{5} \sqrt{1-\frac{16}{3 Q^{2}}}\right) .
$$


Noting that the maximum of $\sigma_{\max }$ in the last form is for $Q=16 / \sqrt{3}$. However, based on the previous equations, $\sigma_{\max }$ is ranging between:

$$
\sigma_{\max }=\pi^{2} \frac{E t h}{l^{2}} \cdot \begin{cases}2 & Q<2, \\ {[2 ; 2.01]} & Q \in[2 ; 2.36[, \\ {[2.01 ; 3.31[} & Q \in[2.36 ; \infty[.\end{cases}
$$

These values of $\sigma_{\max }$ in (47) are calculated when $\Delta$ is ranging between 0 and 2 . Although, the end of deflection can be considered at the second stable position that corresponds to a transversal displacement $\Delta$ lower than 2 . In this case, the new range of $\sigma_{\max }$ is as follows:

$$
\sigma_{\max }=\pi^{2} \frac{E t h}{l^{2}} \cdot\left\{\begin{array}{l}
{[1.93 ; 1.97] Q \in[2.31 ; 2.34]} \\
{[1.97 ; 3.31[Q \in[2.34 ; \infty[}
\end{array}\right.
$$

\subsection{Stresses with high modes}

In the other side, the problem with high modes is complex and hard to handle without approximations. Difficulty lies in the fact that the maximal stress point is difficult to determine analytically.

Axial stress (35) remains the same, while bending stress changes with the consideration of high modes. Bending stress in this case is concluded by introducing (4) into (3) and (3) into (36):

$$
T=\pi^{2} \frac{E z h}{l^{2}}\left[2 \cos 2 \pi X-\sum_{j} A_{j}(j+1)^{2} \cos (j+1) \pi X\right]
$$

The index $j$ in the previous equation refers to $j=1,5,9, \ldots$ for the first kind of solution, and to $j=1,3,5,9, \ldots$ for the third kind of solution.

Drawing equations with changing $\Delta, N$, and $Q$ variables along the beam shows that the midpoint at $x=\frac{l}{2}$ is a local maximum point, and in some cases, the global maximum will not remain at the midpoint but rather a point beside it. However, there are no big difference between stress values at the local and global maximum points. An approximation to suppose the midpoint as the global maximum point is taken. The infinite sum in (49) is calculated by referring to the second sum in (19). The maximal total stress for the first kind of solution is then concluded:

$$
\sigma_{\max }^{N, \Delta}=\pi^{2} \frac{E t h}{l^{2}}\left[\frac{N^{2}}{N^{2}-4 \pi^{2}}+\frac{N^{2}}{12 \pi^{2} Q}+N^{2}\left(\frac{N^{2}}{N^{2}-4 \pi^{2}}-\Delta\right) \frac{\tan \frac{N}{4}}{4 \pi^{2}\left(\frac{N}{4}-\tan \frac{N}{4}\right)}\right] .
$$


The maximum of the stress in the first kind of solution is at the final point. Noting that the final point where $N$ is equivalent to zero is at $\Delta=20 / \pi^{2}$. Introducing these values in (50), the maximal stress becomes:

$$
\sigma_{\max }=\pi^{2} \frac{E t h}{l^{2}} \times \frac{240}{\pi^{4}} .
$$

Idem for the third kind of solution, the maximal total stress can be obtained by setting the value of $N$ and taking into account the constant $A_{3}$ :

$$
\sigma_{\max }^{\Delta}=\pi^{2} \frac{E t h}{l^{2}}\left[\frac{4}{3}+\frac{4}{3 Q}+\frac{4}{9} \sqrt{\frac{-243}{\pi^{2}} \Delta^{2}+\frac{504}{\pi^{2}} \Delta+18-\frac{240}{\pi^{2}}-\frac{108}{Q^{2}}}\right] .
$$

The maximum of the third solution of stress is remarked at $\Delta=28 / 27$. Introducing this $\Delta$ value in (52), we obtain the total maximum stress for the third solution of stress:

$$
\sigma_{\max }=\pi^{2} \frac{E t h}{l^{2}}\left(\frac{4}{3}+\frac{4}{3 Q}+\frac{4}{27} \sqrt{\frac{192}{\pi^{2}}+162-\frac{972}{Q^{2}}}\right) .
$$

We should note here that the global maximum point is at $x=l / 2$ when the deflection $\Delta$ is at the two specific positions taken in (51) and (53). In this context, (51) and (53) are exact.

Based on the above, the maximal stress with considering high modes of buckling is ranging between:

$$
\sigma_{\max }=\pi^{2} \frac{E t h}{l^{2}} \cdot\left\{\begin{array}{l}
2.46 \quad Q<2.42, \\
{[2.46,3.41[Q \in[2.42, \infty[.}
\end{array}\right.
$$

Noting that the maximum for the maximal stress in the third kind of solution is for $Q=\sqrt{\frac{156}{2+\frac{64}{27 \pi^{2}}}}$.

\section{Simulations and Discussion}

In this section, results with and without the approximation of neglecting high modes of buckling, are compared with finite elements simulations using ANSYS.

Simulations are made on a mechanism of two curved beams connected in the middle in order to prevent unsymmetrical buckling modes from occurring. In theory, the snapping force $f$ will be doubled with the number of beams while the deflection $d$ and stresses inside the beam remain the same.

A comparison between the snapping force theory with and without high modes of buckling and simulation of a silicon curved beam with $5 \mathrm{~mm}$ length, $20 \mu \mathrm{m}$ thickness, $10 \mathrm{~mm}$ depth, $80 \mu \mathrm{m}$ height and a Young's modulus of $169 \mathrm{GPa}$ is presented in Fig. 8.

Curves in Fig. 8 show a good agreement between the present theory and FEM simulation. The curve without high modes of buckling was compared previously by Qiu et al. [2004] with FEM simulations and experiments. 


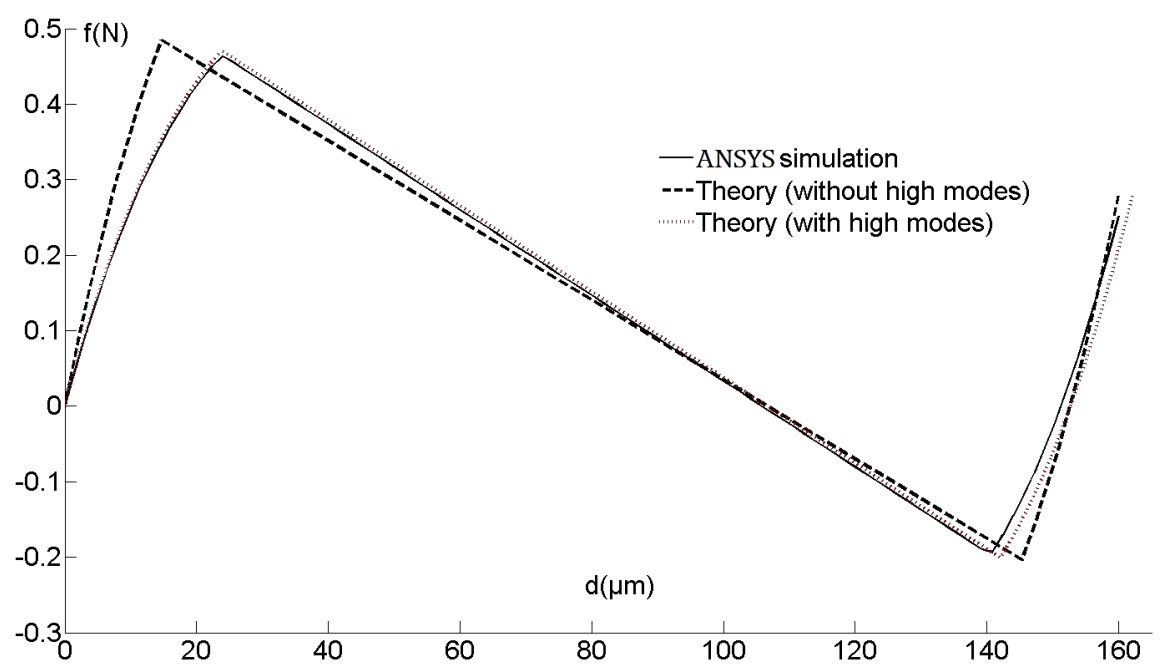

Fig. 8. Comparison of the snapping-force behavior during deflection between theory and FEM simulation.

The presented modeling with high modes shows to be more similar to the FEM simulation in terms of the snapping forces. It allows obtaining the expressions of the different values of $Q$ and the main snapping points $\left(\Delta_{\text {top }}, F_{\text {top }}, \Delta_{\text {bot }}\right.$, $\left.F_{\text {bot }}\right)$ that include the effects of all modes of buckling. In the example given in Fig. 8, the relative difference between the negligence and the consideration of high modes of buckling for the values of the snapping points $\left(\Delta_{\text {top }}, F_{\text {top }}, \Delta_{\text {bot }}, F_{\text {bot }}\right)$ is $(38.53 \%, 2.93 \%, 2.32 \%, 1.39 \%)$, respectively.

The quite large error on the $\Delta_{\text {top }}$ parameter could be a problem for the design of a bistable system. Reminding that the bistable system, as we assume in this paper, is composed of a passive bistable mechanism such a pre-shaped curved beam and actuators for switching between both stable positions. The snapping points in terms of displacements and forces respectively define the stroke and the force that the actuators have to provide. Thus, the accurate knowledge of the relations between the snapping points and the pre-shaped curved beam dimensions is very important in order to achieve the best integration of the complete bistable system.

Noting that the curves in Fig. 8, with and without high modes, meet at the two stable positions.

In the other side, Fig. 9 shows a comparison of the evolution of maximal stresses, between the theory with and without high modes of buckling and simulation of a curved beam with the same dimensions and properties.

The importance of high modes of buckling is more obvious in the calculation of stresses. As shown in Fig. 9, neglecting high modes will make a significant difference between the calculated stress and the simulation. Differences appear in the shape of the stress curves and in the highest stress point position. 


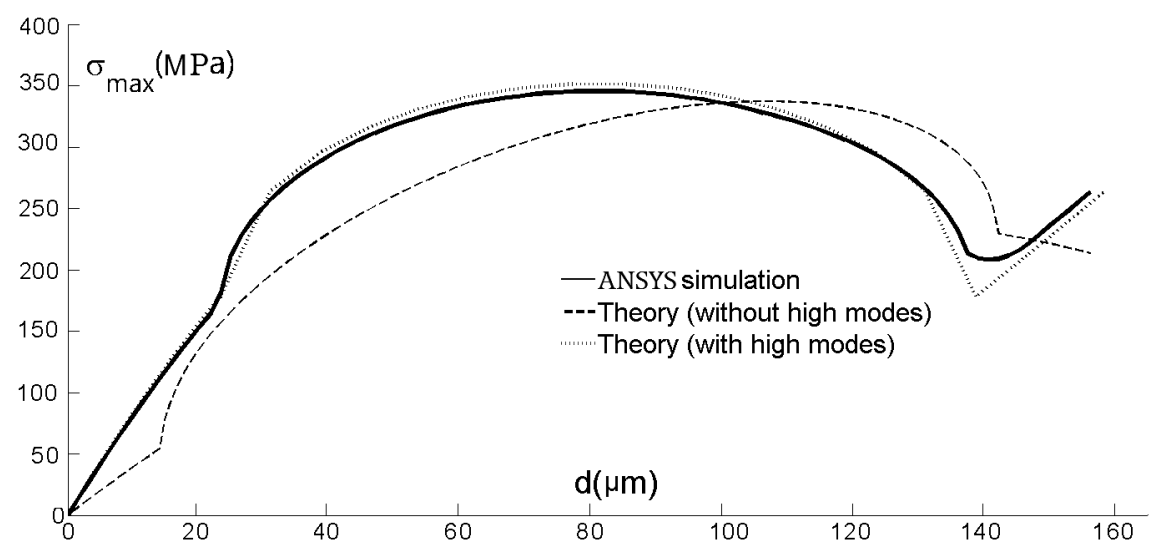

Fig. 9. Comparison between the maximal stress value during deflection between theory and FEM simulation.

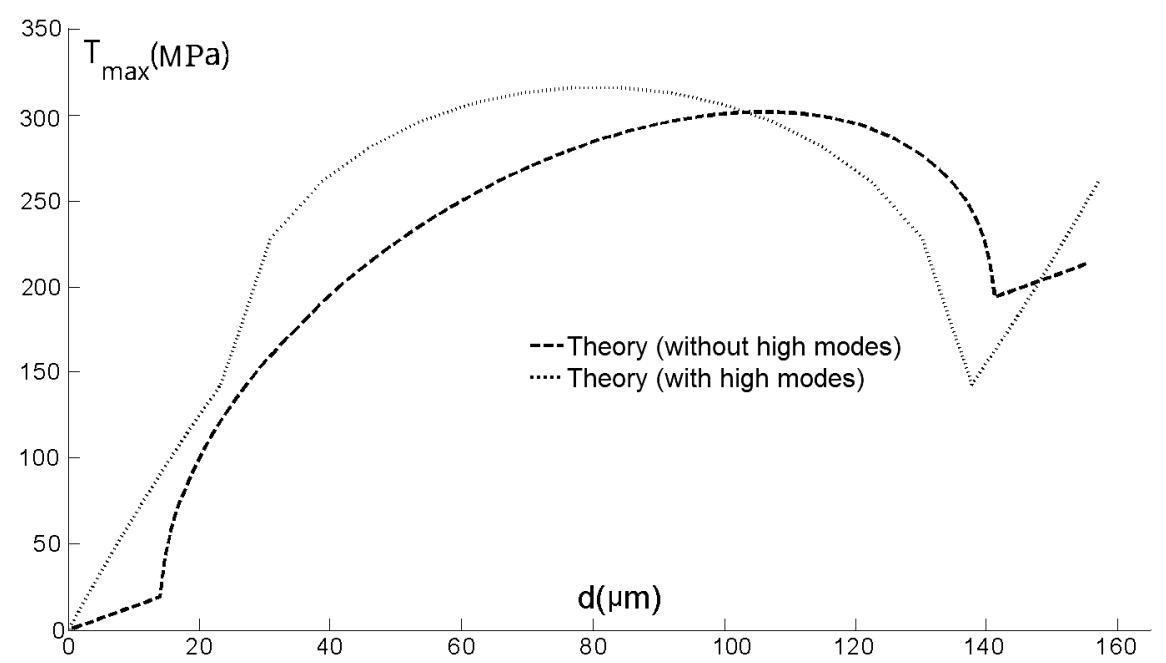

Fig. 10. Comparison between the bending maximal stress value during deflection with and without high modes of buckling.

Figure 10 shows the evolution of the maximal bending stress in the two cases, with and without high modes of buckling.

Comparing the results in Figs. 9 and 10, we conclude that the bending stress has the main contribution in the total stress value. The bending and total stress curves have almost the same shape. The contribution of high modes of buckling is more important in the calculation of the bending stresses.

In the first kind of solutions, the axial stress evolves after deflection from the two sides of buckling to the middle (Fig. 5), while the maximal bending stress is higher when the curved beam is far away from its initial position (Fig. 10). 
In the third kind of solutions, the axial stress is constant along the beam (Fig. 5), while the bending stress has a maximum around the middle of deflection (Fig. 10).

Calculating internal stresses in the curved beam is important for design purposes, particularly for miniaturization and for determining the design limits under elastic or failure limits.

The accurate determination of the relation between the beam dimensions and the stress state allows identifying the limits of miniaturization and avoiding the fracture of a miniature curved beam.

\section{Conclusion}

We investigated the effect of high modes of buckling in the modeling of a pre-shaped curved beam. In a first stage, the presented modeling develops further the snapping forces solution and bistability conditions in order to include high modes of buckling. In a second stage, we developed the analytical solution of the stress inside the beam during deflection between the two sides of buckling.

The buckling with or without mechanical conditions on antisymmetric modes, the force characteristics, bistability conditions and internal stresses have been described in this paper based on mathematical approach in order to provide a clear physical understanding of the nonlinear bistable behavior of a curved beam. Finally, the calculus was compared with and without high modes and with FEM simulations.

The results show that considering high modes of buckling allows improving the accuracy of the snapping force curves, while the importance is more obvious for the calculation of the stress state.

The presented work in this paper constitutes a basis for the design and the optimization of a pre-shaped curved beam bistable mechanism. Future works aim to define the capabilities of miniaturization of such bistable mechanism as a function of the expected mechanical performances and the elastic or failure limits.

\section{Acknowledgment}

This work has been supported by the Labex-ACTION Project (Contract "ANR-11LABX-01-01").

\section{References}

Abadie, J., Chaillet, N. and Lexcellent, C. [2009] "Modeling of a new sma micro-actuator for active endoscopy applications," Mechatronics 19(4), 437-442.

Barth, J. and Kohl, M. [2010] "A bistable magnetically enhanced shape memory microactuator with high blocking forces," Physics Procedia 10, 189-196.

Camescasse, B. [2013] Actionnements statique et dynamique dun mecanisme bistable: aspects modélisation, conception et expérimental, $\mathrm{PhD}$ thesis, Université Pierre et Marie Curie. 
Cazottes, P. [2009] Actionnement des systeèmes bistables: modélisation et études expérimentales, Thèse de doctorat, Université Pierre et Marie Curie, Paris, France.

Chalvet, V., Haddab, Y. and Lutz, P. [2013] "A microfabricated planar digital microrobot for precise positioning based on bistable modules," IEEE Transactions on Robotics 29(3), 641-649.

Chalvet, V., Zarzycki, A., Haddab, Y. and Lutz, P. [2011] "Digital microrobotics based on bistable modules: Design of a non-redundant digital micropositioning robot," in 2011 IEEE International Conference on Robotics and Automation (ICRA), Shanghai, China, pp. 3628-3633, doi: 10.1109/ICRA.2011.5979748.

Chen, G., Aten, Q. T., Zirbel, S., Jensen, B. D. and Howell, L. L. [2009a] "A tristable mechanism configuration employing orthogonal compliant mechanisms," Journal of Mechanisms and Robotics 2(1), 014501, doi: 10.1115/1.4000529.

Chen, G., Gou, Y. and Zhang, A. [2011] "Synthesis of compliant multistable mechanisms through use of a single bistable mechanism," Journal of Mechanical Design 133(8), 081007, doi: 10.1115/1.4004543, http://dx.doi.org/10.1115/1.4004543.

Chen, G., Wilcox, D. L. and Howell, L. L. [2009b] "Fully compliant double tensural tristable micromechanisms (dttm)," Journal of Micromechanics and Microengineering 19(2), 025011 .

Chen, J.-S. and Hung, S.-Y. [2012] "Exact snapping loads of a buckled beam under a midpoint force," Applied Mathematical Modeling 36(4), 1776-1782, doi: 10.1016/j.apm.2011.09.013.

Chen, J.-S. and Tsao, H.-W. [2013] "Static snapping load of a hinged extensible elastica," Applied Mathematical Modeling 37(18), 8401-8408.

Chen, Q., Haddab, Y. and Lutz, P. [2008] "Digital microrobotics based on bistable modules: Design of compliant bistable structures," IEEE/ASME International Conference on Mechtronic and Embedded Systems and Applications, 2008. MESA 2008, Beijing, China, pp. 36-41, doi: 10.1109/MESA.2008.4735711.

Chen, Q., Haddab, Y. and Lutz, P. [2010] "Characterization and control of a monolithically fabricated bistable module for microrobotic applications," 2010 IEEE/RSJ International Conference on Intelligent Robots and Systems (IROS), Taipei, Taiwan, pp. 5756-5761, doi: 10.1109/IROS.2010.5650487.

Chiao, M. and Lin, L. [2000] "Self-buckling of micromachined beams under resistive heating," Journal of Microelectromechanical Systems 9(1), 146-151.

Driesen, M., Ceyssens, F., Decoster, J. and Puers, R. [2010] "Nickel-plated thermal switch with electrostatic latch," Sensors and Actuators A: Physical 164(1), 148-153.

Emam, S. A. and Nayfeh, A. H. [2004] "On the nonlinear dynamics of a buckled beam subjected to a primary-resonance excitation," Nonlinear Dynamics 35(1), 1-17.

Fukuta, Y., Chapuis, Y.-A., Mita, Y. and Fujita, H. [2006] "Design, fabrication, and control of mems-based actuator arrays for air-flow distributed micromanipulation," Journal of Microelectromechanical Systems 15(4), 912-926.

Hafez, M., Lichter, M. and Dubowsky, S. [2003] "Optimized binary modular reconfigurable robotic devices," IEEE/ASME Transactions on Mechatronics 8(1), 18-25, doi: 10.1109/TMECH.2003.809156.

Huang, H.-W. and Yang, Y.-J. [2013] "A mems bistable device with push-on-push-off capability," Journal of Microelectromechanical Systems 22(1), 7-9.

Jensen, B. D., Parkinson, M. B., Kurabayashi, K., Howell, L. L. and Baker, M. S. [2001] "Design optimization of a fully-compliant bistable micro-mechanism," Ann Arbor MI 48109, 2125. 
Krylov, S. and Dick, N. [2010] "Dynamic stability of electrostatically actuated initially curved shallow micro beams," Continuum Mechanics and Thermodynamics 22(6-8), $445-468$.

Nayfeh, A. H. and Emam, S. A. [2008] "Exact solution and stability of postbuckling configurations of beams," Nonlinear Dynamics 54(4), 395-408.

Niu, X., Brochu, P., Stoyanov, H., Yun, S. R. and Pei, Q. [2012] "Bistable electroactive polymer for refreshable braille display with improved actuation stability," SPIE Smart Structures and Materials + Nondestructive Evaluation and Health Monitoring, International Society for Optics and Photonics, pp. 83400R-83400R.

Oberhammer, J., Tang, M., Liu, A.-Q. and Stemme, G. [2006] "Mechanically tri-stable, true single-pole-double-throw (spdt) switches," Journal of Micromechanics and Microengineering 16(11), 2251.

Pane, I. Z. and Asano, T. [2008] "Investigation on bistability and fabrication of bistable prestressed curved beam," Japanese Journal of Applied Physics 47, 5291.

Park, S. and Hah, D. [2008] "Pre-shaped buckled-beam actuators: Theory and experiments," Sensors and Actuators A: Physical 148(1), 186-192.

Qiu, J., Lang, J. H. and Slocum, A. H. [2001] "A centrally-clamped parallel-beam bistable mems mechanism," MEMS 2001. The 14th IEEE International Conference on Micro Electro Mechanical Systems, 2001. (IEEE), pp. 353-356.

Qiu, J., Lang, J. H. and Slocum, A. H. [2004] "A curved-beam bistable mechanism," Journal of Microelectromechanical Systems 13(2), 137-146.

Remmert, R. [1991] "Modes of convergence in function theory," Theory of Complex Functions (Springer), pp. 91-107.

Stoimenov, B. L., Rossiter, J. M. and Mukai, T. [2007] "Manufacturing of ionic polymermetal composites (IPMCs) that can actuate into complex curves," The 14th International Symposium on: Smart Structures and Materials and Nondestructive Evaluation and Health Monitoring, International Society for Optics and Photonics, pp. 65240T-65240T-11.

Tajaddodianfar, F., Yazdi, M. H. and Pishkenari, H. N. [2014] "Dynamics of bistable initially curved shallow microbeams: Effects of the electrostatic fringing fields," IEEE/ASME International Conference on Advanced Intelligent Mechatronics AIM, IEEE/ASME, pp. 1279-1283.

Timoshenko, S. [1961] Theory of Elastic Stability (McGraw-Hill).

Vangbo, M. [1998] "An analytical analysis of a compressed bistable buckled beam," Sensors and Actuators A: Physical 69(3), 212-216.

Wu, Y., Ding, G., Zhang, C., Wang, J., Mao, S. and Wang, H. [2010] "Design and implementation of a bistable microcantilever actuator for magnetostatic latching relay," Microelectronics Journal 41(6), 325-330.

Yu, Z., Niu, X., Brochu, P., Yuan, W., Li, H., Chen, B. and Pei, Q. [2010] "Bistable electroactive polymers (BSEP): Large-strain actuation of rigid polymers," SPIE Smart Structures and Materials + Nondestructive Evaluation and Health Monitoring, International Society for Optics and Photonics, pp. 76420C76420C-9.

Zaidi, S. S. H., Cherfi-Boulanger, Z. and Lamarque, F. [2011] Méthode d'apport d'énergie sélective et sans contact: applications aux micro-actionneurs bistables, Thèse doctorat, Université de Technologie de Compiègne, France.

Zhang, Y.-H., Ding, G., Shun, X., Gu, D., Cai, B. and Lai, Z. [2007] "Preparing of a high speed bistable electromagnetic rf mems switch," Sensors and Actuators A: Physical 134(2), 532-537. 